IASSNS-HEP-93-31

SWAT-93-01

May 1993

\title{
String-Based Methods in Perturbative Gravity
}

\author{
Zvi Bern* \\ Institute for Advanced Study \\ Olden Lane \\ Princeton, NJ 08540 \\ David C. Dunbar \\ Department of Physics \\ University College of Swansea \\ Swansea SA2 8PP, U.K. \\ and \\ Tokuzo Shimada \\ Natural Science Division \\ Izumi Campus, Meiji University \\ Eifuku 1-9-1, Suginami, Tokyo 168, Japan
}

\begin{abstract}
String theory implies a relatively modest growth in computational complexity for perturbative gravity calculations as compared to gauge theory calculations, contrary to field theory expectations. An explicit string-based calculation, which would be extremely difficult using conventional techniques, is presented to illustrate this.
\end{abstract}

\footnotetext{
* Permanent address: Dept. of Physics, UCLA, LA, CA 90024
} 
1. Introduction. Perturbative computations in gravity are notorious for their algebraic complexity, being many orders of magnitude more complicated than the corresponding gauge theory computations. For example, a brute force computation of the one-loop four-graviton scattering amplitude using conventional Feynman diagram techniques [1] involves $\sim 10^{8}$ terms. Even with the background field method [2] in a brute force computation one would encounter $\sim 10^{6}$ terms. The size of these intermediate expressions may be compared to the final results which are quite compact; indeed the amplitude for one minus and three plus helicities fits on a line.

Recently, a string-based technique significantly more efficient than conventional Feynman diagram techniques was developed for the computation of one-loop $n$ gluon amplitudes [3-7]. Obvious questions are whether this technique can be extended to other cases and whether string theory provides additional non-trivial guidance for these extensions. In this letter, we address these questions by extending these string-based techniques to perturbative gravity. At tree level, Berends, Giele and Kuijf [8] have already used string theory [9] to give compact expressions for a class of tree-level gravity amplitudes using known Yang-Mills tree amplitudes [10]. For $N=8$ supergravity, Green, Schwarz and Brink have used the Green-Schwarz formulation of string theory to give compact results for the four graviton one-loop amplitude [11].

The string-based technique was originally developed to compute one-loop gluon matrix-elements that are formidable to compute but which are required for current and future experiments. This technology was a key ingredient in the first calculation of the one-loop five-gluon amplitude (which will enter into the analysis of three-jet events at hadron colliders) [6]. This technique has been summarized in terms of systematic rules $[4,5]$ for the one-loop $n$-gluon amplitude which require no knowledge of string theory and bypass much of the algebra associated with Feynman diagram calculations.

To convert the rules to the case of one-loop graviton scattering amplitudes we alter the details of the string construction to recover gravity amplitudes rather than gauge theory amplitudes in the infinite string tension limit [12]. Since the string based rules for gauge theories are already computationally efficient one expects considerable advantages in using string-based rules for gravity.

Calculations of one-loop gravity amplitudes have never been performed using traditional Feynman diagram methods. With the string-based method $[4,5]$ we exhibit a four-point graviton-by-graviton scattering calculation for a particular helicity configuration and arbitrary particle content. This computation would be exceedingly difficult by traditional Feynman diagram techniques but is very simple with string based techniques. 
Given the conventional field theory understanding of the efficiency of the stringbased methods when performing a one-loop Yang-Mills calculation [13], one might think to apply this knowledge to other cases without further appeals to string theory [14]. However, for the case of one-loop gravity the field theory understanding of Yang-Mills is insufficient to obtain the full benefit of the string-based methods. In particular, the structure of the string integrand is

$$
(\text { Closed String }) \sim(\text { Open String })^{2}
$$

Since closed strings contain gravity and open strings contain gauge theory there should be a formulation of gravity with the property that the integrands of the diagrams satisfy

$$
(\text { Gravity }) \sim(\text { Yang-Mills })^{2} \text {. }
$$

In string theory this relationship can be made precise.

Given that string theory has this property one can attempt to reorganize field theory to mimic this. To do so non-trivial field redefinitions and gauge choices are required. In this way one can attempt to mimic the string simplicity of the amplitude, but in a conventional field theory approach there is no guiding principle. (The field theory first quantized formalism $[15,16]$ could be used for studying the effective action.)

2. Field theory structure. We will now examine the properties that a reorganization of conventional field theory must satisfy to mimic the string-based structure (1). The starting point in field theory is the Einstein-Hilbert action

$$
S[h]=\frac{2}{\kappa^{2}} \int d^{4} x \sqrt{-g} R .
$$

Our conventions are chosen so that the kinetic term has the correct canonical normalization. The metric is expanded as $g_{\mu \nu}=\eta_{\mu \nu}+\kappa h_{\mu \nu}$ where $h_{\mu \nu}$ is the graviton field. The first step in finding a conventional field theory formulation which mimics string theory is to find a suitable propagator for $h_{\mu \nu}$. In string theory the propagator $\left(L_{0}+\tilde{L}_{0}-2\right)^{-1}$, where $L_{0}$ and $\tilde{L}_{0}$ are left- and right-mover world-sheet Hamiltonians, does not contain Lorentz indices. This indicates that the required field theory propagator should have a trivial Lorentz structure and therefore be proportional to the unit tensor

$$
I_{\mu \nu ; \rho \sigma}=\frac{1}{2}\left(\eta_{\mu \rho} \eta_{\nu \sigma}+\eta_{\mu \sigma} \eta_{\nu \rho}\right)
$$

The unit tensor is a symmetrization of a product of $\eta_{\mu \nu}$ 's which is the tensor in the propagator of Feynman-like gauges in Yang-Mills. The commonly used de Donder 
gauge gravity propagator $[1]$ is

$$
P_{\mu \nu ; \rho \sigma}=i\left[\frac{I_{\mu \nu ; \rho \sigma}}{p^{2}+i \varepsilon}-\frac{1}{D-2} \frac{\eta_{\mu \nu} \eta_{\rho \sigma}}{p^{2}+i \varepsilon}\right]
$$

where the signature of $g_{\mu \nu}$ is $(+,-,-,-)$. This propagator is not of the desired form, since there is an extra trace piece and so the de Donder gauge is not an appropriate candidate to mimic the string organization. Although it is not possible to obtain a propagator with only a unit tensor within the class of standard gauges, since the de Donder gauge propagator is close to the desired form one might suspect that there exists a modification of the theory with the desired field theory propagator. String theory suggests a natural way of accomplishing this.

In string theory there is always an additional field associated with the graviton - the dilaton $\varphi$. This suggests that one can add a dilaton to the theory in order to produce a simple propagator to aid in calculations. At the end of a calculation one would subtract out the dilaton contribution, which is quite simple because it is a scalar. In a string-based calculation one also needs to subtract the dilaton contribution. In string theory there is in addition an antisymmetric tensor which must be subtracted; in four dimensions, this is effectively another scalar.

From the field theory understanding of the gauge theory rules [13], the background field method [2] is needed to mimic the loop part of the string-based rules. Consider the one-loop effective action of gravity coupled to a dilaton and carry out a background field expansion $g_{\mu \nu}=\bar{g}_{\mu \nu}+\kappa h_{\mu \nu}$. With the background field de Donder gauge choice, the part of the action quadratic in the quantum fields is

$$
S=\int d^{4} x \sqrt{-\bar{g}}\left[-\frac{1}{2} h_{\mu \nu} D^{2} h^{\mu \nu}+h^{\mu \nu} R_{\mu \rho \nu \sigma} h^{\rho \sigma}+\frac{1}{4} h_{\mu}^{\mu} D^{2} h_{\mu}^{\mu}-\frac{1}{2} \varphi D^{2} \varphi-\bar{\chi}^{\mu} D^{2} \chi_{\mu}\right]
$$

where we have used the on-shell conditions on the background field and have included the ghosts $\chi_{\mu}$. The curvature and covariant derivatives are with respect to the background field. Consider the field redefinition

$$
h_{\mu \nu}=\tilde{h}_{\mu \nu}+\frac{\eta_{\mu \nu}}{\sqrt{D-2}} \tilde{\varphi} \quad ; \quad \varphi=\frac{1}{\sqrt{2}} \tilde{h}_{\mu}^{\mu}+\sqrt{\frac{D-2}{2}} \tilde{\varphi} .
$$

This has no effect on the value of the effective action since it is only a change of variables for the internal quantum field. (There is a trivial Jacobian in the path integral which is unity in dimensional regularization.) Performing the field redefinition yields

$$
S=\int d^{4} x \sqrt{-\bar{g}}\left[-\frac{1}{2} \tilde{h}_{\mu \nu} D^{2} \tilde{h}^{\mu \nu}+\tilde{h}^{\mu \nu} R_{\mu \rho \nu \sigma} \tilde{h}^{\rho \sigma}+\frac{1}{2} \tilde{\varphi} D^{2} \tilde{\varphi}-\bar{\chi}^{\mu} D^{2} \chi_{\mu}\right]
$$


where again we have dropped terms that vanish after imposing the equation of motion on the background field $\bar{g}$. In this action the 'graviton' propagator is proportional to the unit tensor and is thus of the required form to mimic the string organization. Furthermore, the background field graviton three vertex $G_{3}$ derived from this action is

$$
G_{3 \mu \nu \rho}^{\kappa \lambda \delta}(k, p, q)=-\frac{i}{8} \kappa\left[V_{3 \mu \nu \rho}(k, p, q) \times V_{3}^{\kappa \lambda \delta}(k, p, q)+\{\mu \leftrightarrow \kappa\},\{\nu \leftrightarrow \lambda\},\{\rho \leftrightarrow \delta\}\right]
$$

where $V_{3}$ is proportional to the kinematic part of the Feynman gauge background field Yang-Mills three-vertex,

$$
V_{3 \mu \nu \rho}(k, p, q)=\eta_{\nu \rho} \frac{(p-q)_{\mu}}{2}-\eta_{\mu \rho} k_{\nu}+\eta_{\mu \nu} k_{\rho}
$$

where $k$ is the momentum of the on-shell background field line and $p$ and $q$ are the momenta of the internal lines. The vertex $G_{3}$ is therefore of the desired form to mimic string theory since it is composed of products of Yang-Mills vertices.

In background field method one would sew tree diagrams in some other gauge onto the one particle irreducible diagrams [2]. For the tree parts of diagrams, the relevant gauge choices and field redefinitions necessary to mimic the string form are more complicated but are similar (although not identical) to the choices made by van de Ven [17] in his computation of the two-loop infinity of gravity. A field redefinition is also needed to remove the trace term in the tree-level graviton propagator.

One can expect that this process of reformulating field theory to mimic the stringbased structure can be continued, but the process becomes increasingly obscure. For example, Yang-Mills only has three- and four-point vertices while gravity has infinitely many vertices. ${ }^{*}$ A simpler approach to carry out calculations is to proceed directly using string theory. The procedure for obtaining field theory rules from string theory has been described in refs. $[4,18]$.

3. One-loop rules for gravity. The one-loop string-based rules for gravity are similar to those for gauge theory [4] so we only outline the differences between the two sets of rules. We use the bosonic string form of the rules [18,19] since the kinematic expression is simpler than the heterotic string form originally used [4,5] although it contains identical information. (The heterotic string was used in the original derivation of the rules because of its full consistency.)

\footnotetext{
* In the field theory limit of string theory higher point vertices appear from a combination of $\delta$-functions in the Schwinger proper time and by cancellation of kinematic poles against factors in the kinematic expression.
} 
The starting point of these rules are labeled $\phi^{3}$ diagrams (excluding tadpoles.) Considering that gravity has an infinite set of higher point Feynman vertices, a description in terms of $\phi^{3}$ diagrams may seem surprising, but the contributions from all such higher vertices are implicitly included. There is no need to consider diagrams with loops isolated on external legs as these vanish in dimensional regularization.

The external legs of the diagrams should be labeled in the same way as ordinary Feynman diagrams with all orderings included. This is unlike the gauge theory case where the legs were color ordered. The inner lines of a tree attached to a loop are labeled according to the rule that as one moves from the outer lines to the inner lines, one labels the inner line by the label of the most clockwise of the two outer lines. (See refs. $[4,5,19]$ for further details.) According to the rules, each labeled $n$-point $\phi^{3}$-like diagram evaluates to

$$
\begin{aligned}
\mathcal{D}=i \frac{(-\kappa)^{n}}{(4 \pi)^{2-\epsilon / 2}} \Gamma\left(n_{\ell}-2+\epsilon / 2\right) & \int_{0}^{1} d x_{i_{n_{\ell}-1}} \int_{0}^{x_{i_{n_{\ell}-1}}} d x_{i_{n_{\ell}-2}} \cdots \int_{0}^{x_{i_{3}}} d x_{i_{2}} \int_{0}^{x_{i_{2}}} d x_{i_{1}} \\
& \times \frac{K_{\mathrm{red}}}{\left(\sum_{l<m}^{n_{\ell}} P_{i_{l}} \cdot P_{i_{m}} x_{i_{m} i_{l}}\left(1-x_{i_{m} i_{l}}\right)\right)^{n_{\ell}-2+\epsilon / 2}}
\end{aligned}
$$

where the ordering of the loop parameter integrals corresponds to the ordering of the $n_{\ell}$ lines attached to the loop, $x_{i j} \equiv x_{i}-x_{j}$, and $K_{\text {red }}$ is the reduced kinematic factor. The string-based rules efficiently yield $K_{\text {red }}$ in a compact form. The lines attached to the loop carry momenta $P_{i}$ which will be off-shell if there is a tree attached to that line. The dimensional regularization parameter $\epsilon=4-D$ handles all ultraviolet and infrared divergences. The $x_{i_{m}}$ are related to ordinary Feynman parameters by $x_{i_{m}}=\sum_{j=1}^{m} a_{j}$. The amplitude is then given by summing over all diagrams.

The starting point for evaluating $K_{\text {red }}$ for any diagram is the graviton kinematic expression

$$
\begin{aligned}
\mathcal{K}=\int \prod_{i=1}^{n} d x_{i} d \bar{x}_{i} \prod_{i<j}^{n} \exp \left[k_{i} \cdot k_{j} G_{B}^{i j}\right] \exp \left[\left(k_{i} \cdot \varepsilon_{j}-k_{j} \cdot \varepsilon_{i}\right) \dot{G}_{B}^{i j}-\varepsilon_{i} \cdot \varepsilon_{j} \ddot{G}_{B}^{i j}\right] \\
\quad \times\left.\exp \left[\left(k_{i} \cdot \bar{\varepsilon}_{j}-k_{j} \cdot \bar{\varepsilon}_{i}\right) \dot{\bar{G}}_{B}^{i j}-\bar{\varepsilon}_{i} \cdot \bar{\varepsilon}_{j} \ddot{G}_{B}^{i j}\right]\right|_{\text {multi-linear }}
\end{aligned}
$$

where the 'multi-linear' indicates that only the terms linear in all $\varepsilon_{i}$ and $\bar{\varepsilon}_{i}$ are included. The graviton polarization tensor is reconstructed by taking $\varepsilon_{i}^{\mu} \bar{\varepsilon}_{i}^{\nu} \rightarrow \varepsilon_{i}^{\mu \nu}$. This kinematic expression is obtained from a bosonic string and contains the same information as that obtained from a superstring [18]. The structure of this kinematic expression is that the 
polarization factors are a product of two gauge theory factors, $[4,5,18]$ corresponding to the left- and right-movers of the underlying closed string theory. In string theory the $G_{B}$ are Green functions on the world sheet, but in the field theory limit these become 'Feynman parameter functions'. From a conventional Feynman diagram point of view, the existence of a universal kinematic function is surprising as there is apparently no simple relationship between the various Feynman diagrams contributing to a given process.

In the form of the rules presented in refs. [4,18], one integrates by parts to remove all $\ddot{G}_{B}$. In the case of gravity the integration by parts on the left and right are not independent and certain cross-terms where a left-mover derivative hits right-mover terms must be taken into account. We will discuss these cross-terms elsewhere since the integration by parts is not necessary for the calculation in the next section. It is this integration by parts step which reduces gravity to a $\phi^{3}$ structure. (This step is not an essential part of the string-based method, which can be formulated without integration by parts [7].)

Given the integrated by parts kinematic expression, for a particular diagram with a two-point tree with lines labeled by $i$ and $j$, with $i$ appearing before $j$ in the clockwise ordering, the tree rules tell one to replace a $\left(\dot{G}_{B}^{i j}\right)^{n}\left(\dot{\bar{G}}_{B}^{i j}\right)^{m}$ in each term by a factor of $\delta_{n, 1} \delta_{m, 1}\left(-2 k_{i} \cdot k_{j}\right)^{-1}$. One moves from the outside inward iteratively, replacing the functions as described. These tree rules do not depend on what particles circulate in the loop and are similar to those in refs. $[4,5,19]$.

After the tree rules are applied to a given diagram one then applies loop substitution rules. These are essentially identical rules as for Yang-Mills applied independently to both the left- and right-mover parts of the kinematic expression. This provides an explicit diagram-by-diagram relationship between the one-loop gravity amplitude and the corresponding gauge theory amplitude. For gravitons (and the associated ghosts) circulating in the loop, every term generates two types of contributions.

The first contribution for left-movers is obtained by multiplying the kinematic expression by an overall factor of $\left(2-\epsilon \delta_{R}\right)$ and substituting

$$
\dot{G}_{B}^{i j} \longrightarrow \frac{1}{2}\left(-\operatorname{sign}\left(x_{i j}\right)+2 x_{i j}\right)
$$

and exactly the same substitution for the right-mover $\dot{\bar{G}}_{B}^{i j}$. The parameter $\delta_{R}$ depends on the precise form of the regularization scheme used [4]. When this first type of term occurs for both left- and right-movers instead of a factor of $\left(2-\epsilon \delta_{R}\right)^{2}$, the correct factor is $\left(4-\epsilon \delta_{R}\right)\left(1-\epsilon \delta_{R}\right) / 2$, which is the number of graviton degrees of freedom. More generally for a theory of gravity containing various particle types, the factor $\left(2-\epsilon \delta_{R}\right)^{2}$ 
is replaced by

$$
N_{s}=N_{b}-N_{f}
$$

where $N_{b}$ is the number of bosonic states (including any modifications due to dimensional regularization) and $N_{f}$ is the number of fermionic states which circulate in the loop.

The second type of contribution for gravitons arises if a particular term contains a cycle of $\dot{G}_{B} \mathrm{~S}[18,19]$. The rules for cycle contributions are essentially the same as for gauge theory except that now there are both left and right contributions. For the graviton in the loop one simply takes the gauge theory vector rules on the right and on the left.

For other particles in the loop one applies rules appropriate for the particle under consideration. For example, a contribution from a gravitino in the loop can be obtained by using gauge theory vector loop rules on the left and fermion loop rules on the right. In this way the contribution of gravitons, gravitinos, vectors, fermions or scalars to the one-loop gravity amplitudes can be obtained by independently choosing gauge theory scalars, fermions, or vector loop rules given in refs. $[18,19]$ for the left and right pieces.

Modifications to include masses for the internal fermions or scalars is simple; the only change is in the denominator in eq. (3) where the massless Feynman denominator is replaced with one corresponding to massive states circulating in the loop.

To illustrate the gravity rules we now turn to an explicit example.

4. Sample calculation. We now calculate the $\mathcal{A}\left(1^{-}, 2^{+}, 3^{+}, 4^{+}\right)$four-graviton helicity amplitude. From a conventional Feynman diagram point of view, this computation requires a total of 12 distinct diagrams or 54 diagrams including permutations of external legs. Since gravity vertices contain many terms this would be an extremely difficult calculation with conventional Feynman diagram techniques; using string-based rules we show that this calculation is in fact very easy.

The first step is to insert spinor helicity simplifications into the kinematic expression (4). The spinor helicity method for gravitons $[8,20]$ is related to that for vectors [21] by

$$
\varepsilon^{++}=\varepsilon^{+} \bar{\varepsilon}^{+}, \quad \varepsilon^{--}=\varepsilon^{-} \bar{\varepsilon}^{-}
$$

where $\varepsilon^{ \pm \pm}$are the graviton helicity polarizations and $\varepsilon^{ \pm}$are the vector helicity polarizations defined by $\mathrm{Xu}$, Zhang and Chang. We use the notation for spinor inner products $\left\langle k_{1}^{-} \mid k_{2}^{+}\right\rangle=\langle 12\rangle$ and $\left\langle k_{1}^{+} \mid k_{2}^{-}\right\rangle=[12]$. Using the same choice of spinor helicity reference momenta as in the Yang-Mills computation of ref. [5] simplifies the kinematic 
coefficient to

$$
\begin{aligned}
K= & S\left(\dot{G}_{B}^{13}-\dot{G}_{B}^{12}\right)\left(\dot{G}_{B}^{24}-\dot{G}_{B}^{23}\right)\left(\dot{G}_{B}^{34}+\dot{G}_{B}^{23}\right)\left(\dot{G}_{B}^{34}-\dot{G}_{B}^{24}\right) \\
& \times\left(\dot{\bar{G}}_{B}^{13}-\dot{G}_{B}^{12}\right)\left(\dot{\bar{G}}_{B}^{24}-\dot{\bar{G}}_{B}^{23}\right)\left(\dot{\bar{G}}_{B}^{34}+\dot{\bar{G}}_{B}^{23}\right)\left(\dot{\bar{G}}_{B}^{34}-\dot{\bar{G}}_{B}^{24}\right)
\end{aligned}
$$

where

$$
S=\left(\frac{s^{2} t}{4}\right)^{2}\left(\frac{[24]^{2}}{[12]\langle 23\rangle\langle 34\rangle[41]}\right)^{2}
$$

and the Mandelstam variables are $s=2 k_{1} \cdot k_{2}, t=2 k_{1} \cdot k_{4}$ and $u=-s-t$. Due to the special helicity configuration, $\ddot{G}_{B} \mathrm{~s}$ do not appear and there is therefore no need to integrate by parts.

The next step is to determine which diagrams vanish trivially by the tree rules. There are a total of twelve $\phi^{3}$-like diagrams. Of these, seven vanish by the tree rules. For example, a diagram containing a 1-4 tree vanishes because there are no $\dot{G}_{B}^{14}$ Green functions. Other diagrams which contain a $2-3$ tree vanish because the remaining factors vanish after setting the labels of the two pinched legs together; in this case $\left(\dot{G}_{B}^{34}-\dot{G}_{B}^{24}\right) \rightarrow 0$ for $2 \rightarrow 3$. The only non-vanishing diagrams are the five shown in 1a-e.

First consider 1a. This diagram has no trees so we immediately apply the loop rules. It is not difficult to check that all cycle contributions of the loop cancel amongst themselves whether fermion or vector rules are applied to the right- or left-movers. Thus the reduced kinematic expression can be obtained by applying the substitution rule (5) and multiplying by the number of states $N_{s}$ yielding the Feynman parameter polynomial

$$
N_{s} S x_{2}^{2}\left(1-x_{3}\right)^{2}\left(x_{3}-x_{2}\right)^{4} .
$$

Up to an overall constant this is precisely the square of the Yang-Mills Feynman parameter polynomial for the corresponding diagram derived in refs. $[5,19]$. Inserting this into the loop integral yields

$$
D_{a}=\frac{i \kappa^{4}}{(4 \pi)^{2}} N_{s} S \int_{0}^{1} d x_{3} \int_{0}^{x_{3}} d x_{2} \int_{0}^{x_{2}} d x_{1} \frac{x_{2}^{2}\left(1-x_{3}\right)^{2}\left(x_{3}-x_{2}\right)^{4}}{\left[s x_{1}\left(x_{3}-x_{2}\right)+t\left(x_{2}-x_{1}\right)\left(1-x_{3}\right)\right]^{2}} .
$$

Since this and all following integrals are finite we have set the dimensional regularization parameter $\epsilon$ to zero. This integral is easy to evaluate as the numerator cancels the denominator after performing the $x_{1}$ integral. Diagrams $1 \mathrm{~b}$ and $1 \mathrm{c}$ are just as easy to evaluate. The three contributions are

$$
D_{a}=\frac{i \kappa^{4}}{(4 \pi)^{2}} \frac{N_{s} S}{840 s t}, \quad D_{b}=\frac{i \kappa^{4}}{(4 \pi)^{2}} \frac{N_{s} S}{840 u t}, \quad D_{c}=\frac{i \kappa^{4}}{(4 \pi)^{2}} \frac{N_{s} S}{252 s u} .
$$


This takes care of the box diagrams.

Now we evaluate the two triangle diagrams. First consider 1d. Applying the rules for a 1-2 tree reduces the kinematic coefficient (4) to

$$
K=-\frac{1}{s} S\left(\dot{G}_{B}^{24}-\dot{G}_{B}^{23}\right)\left(\dot{G}_{B}^{34}+\dot{G}_{B}^{23}\right)\left(\dot{G}_{B}^{34}-\dot{G}_{B}^{24}\right)\left(\dot{G}_{B}^{24}-\dot{G}_{B}^{23}\right)\left(\dot{\bar{G}}_{B}^{34}+\dot{\bar{G}}_{B}^{23}\right)\left(\dot{\bar{G}}_{B}^{34}-\dot{\bar{G}}_{B}^{24}\right) .
$$

Applying the loop substitution rule yields the loop integral

$$
D_{d}=-i \frac{\kappa^{4}}{(4 \pi)^{2}} \frac{N_{s} S}{s} \int_{0}^{1} d x_{3} \int_{0}^{x_{3}} d x_{2} \frac{\left(1-x_{3}\right)^{2} x_{2}^{2}\left(x_{3}-x_{2}\right)^{2}}{s x_{2}\left(x_{2}-x_{3}\right)}
$$

which is a trivial integral since the denominator cancels against the numerator. The last non-zero diagram $1 \mathrm{e}$ is similar to evaluate and the two diagrams are

$$
D_{d}=\frac{i \kappa^{4}}{(4 \pi)^{2}} \frac{N_{s} S}{360 s^{2}}, \quad D_{e}=\frac{i \kappa^{4}}{(4 \pi)^{2}} \frac{N_{s} S}{360 u^{2}} .
$$

Summing over all diagrams we have the four-graviton amplitude in a theory with any particle content as

$$
\mathcal{A}\left(1^{-}, 2^{+}, 3^{+}, 4^{+}\right)=\frac{i \kappa^{4}}{(4 \pi)^{2}} \frac{N_{s}}{5760} \frac{s^{2} t^{2}}{u^{2}}\left(u^{2}-s t\right)\left(\frac{[24]^{2}}{[12]\langle 23\rangle\langle 34\rangle[41]}\right)^{2} .
$$

For pure gravity $N_{s}=2$ because the graviton has two helicity states. It is easy to verify that this amplitude has the required crossing symmetry under the interchange of legs 2 , 3 and 4. In a supergravity theory with equal numbers of bosonic and fermionic states $N_{s}=0$ so the amplitude vanishes in agreement with the supersymmetry identities [22]. Note that in this string-based calculation this identity holds at the level of the integrand.

The helicity conserving process $\mathcal{A}\left(1^{-}, 2^{-}, 3^{+}, 4^{+}\right)$is more difficult to compute since cycle contributions no longer vanish and it is infrared divergent. However, even this is relatively easy to compute using the string-based methods.

These calculations may be compared to the corresponding QED calculation of light-by-light scattering. One has a few more diagrams and more complicated Feynman parameter polynomials to integrate, but the extra complication is very slight when compared to the traditional field theory expectation that gravity computations are exceedingly more complicated than QED ones.

In conclusion, gravity provides a further example of how string-based methods can be used to obtain results which would be extremely difficult to obtain using field theory methods. We expect new methods based on string theory to have further non-trivial applications to field theory calculations.

We thank Lance Dixon and David Kosower for helpful discussions. This work was supported by a S.E.R.C. advanced fellowship, NATO grant CRG-910285 and by the Texas National Research Commission grant FCFY9202. 


\section{References}

[1] B.S. DeWitt, Phys. Rev. 162:1239 (1967);

M. Veltman, in Les Houches 1975, Methods in Field Theory, ed R. Balian and J. Zinn-Justin, (North Holland, Amsterdam, 1976).

[2] G. 't Hooft, Acta Universitatis Wratislavensis no. 38, 12th Winter School of Theoretical Physics in Karpacz; Functional and Probabilistic Methods in Quantum Field Theory, Vol. 1 (1975);

B.S. DeWitt, in Quantum Gravity II, eds. C. Isham, R. Penrose and D. Sciama (Oxford, 1981);

L.F. Abbott, Nucl. Phys. B185:189 (1981);

L.F Abbott, M.T. Grisaru and R.K. Schaeffer, Nucl. Phys. B229:372 (1983).

[3] Z. Bern and D.A. Kosower, Phys. Rev. Lett. 66:1669 (1991).

[4] Z. Bern and D.A. Kosower, Nucl. Phys. B379:451 (1992).

[5] Z. Bern and D.A. Kosower, in Proceedings of the PASCOS-91 Symposium, eds. P. Nath and S. Reucroft.

[6] Z. Bern, L. Dixon and D.A. Kosower, Phys. Rev. Lett. 70:2677 (1993).

[7] Z. Bern, L. Dixon and D.A. Kosower, in preparation.

[8] F.A. Berends, W.T. Giele and H. Kuijf, Phys. Lett. 211B:91 (1988).

[9] H. Kawai, D.C. Lewellen and S.-H.H. Tye, Nucl. Phys. B269:1 (1986).

[10] S.J. Parke and T.R. Taylor, Phys. Rev. Lett.56:2459 (1986).

[11] M.B. Green, J.H. Schwarz and L. Brink, Nucl. Phys. B198:472 (1982).

[12] J. Scherk, Nucl. Phys. B31:222 (1971);

A. Neveu and J. Scherk, Nucl. Phys. B36:155 (1972);

J. Minahan, Nucl. Phys. B298:36 (1988).

[13] Z. Bern and D.C. Dunbar, Nucl. Phys. B379:562 (1992).

[14] C.S. Lam, preprints McGill/92-32 and 92/53.

[15] L. Brink, P. Di Vecchia and P. Howe, Phys. Lett. 65B Nucl. Phys. B118 (1977) 76 ;

F.A. Berezin and M.S. Marinov, JETP Lett. 21:320 (1975);

R.Casalbouni, Nouvo Cimento 33A:389 (1976);

M.B. Halpern and P. Senjanovic, Phys. Rev. D15:1655 (1977);

M.B. Halpern, A. Jevicki and P. Senjanovic, Phys. Rev. D16:2476 (1977);

E.S. Fradkin and A.A. Tseytlin, Phys. Lett. 158B:316 (1985); 163B:123 (1985);

Nucl. Phys. B261:1 (1985).

[16] M. Strassler, Nucl. Phys. B385:145 (1992).

[17] A. van de Ven, Nucl. Phys. B378:309 (1992). 
[18] Z. Bern, Phys. Lett. 296B:85 (1992).

[19] Z. Bern, UCLA/93/TEP/5, hep-ph/9304249, procceedings of TASI 1992.

[20] H.T. Cho, K.L. Ng, Phys. Rev. D47:1692 (1993).

[21] F. A. Berends, R. Kleiss, P. De Causmaecker, R. Gastmans and T. T. Wu, Phys. Lett. 103B:124 (1981);

P. De Causmaeker, R. Gastmans, W. Troost and T. T. Wu, Nucl. Phys. B206:53 (1982);

R. Kleiss and W. J. Stirling, Nucl. Phys. B262:235 (1985);

J. F. Gunion and Z. Kunszt, Phys. Lett. 161B:333 (1985);

R. Gastmans and T.T. Wu, The Ubiquitous Photon: Helicity Method for QED and QCD (Clarendon Press) (1990);

Z. Xu, D.-H. Zhang and L. Chang, Nucl. Phys. B291:392 (1987).

[22] M.T. Grisaru, H.N. Pendleton and P. van Nieuwenhuizen, Phys. Rev. D15:996 (1977) ;

M.L. Mangano and S.J. Parke, Phys. Rep. 200:301 (1991). 


\section{Figure Caption}

Fig 1: The diagrams which do not vanish after applying the tree rules. 


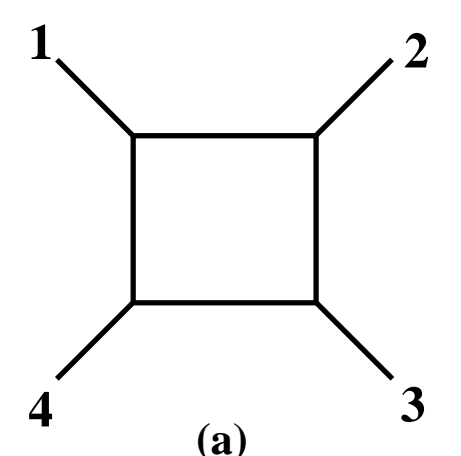

(a)

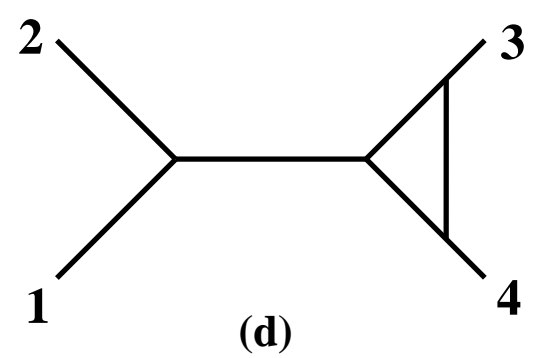

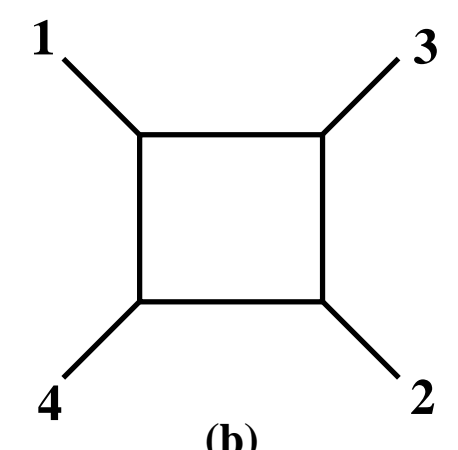

(b)
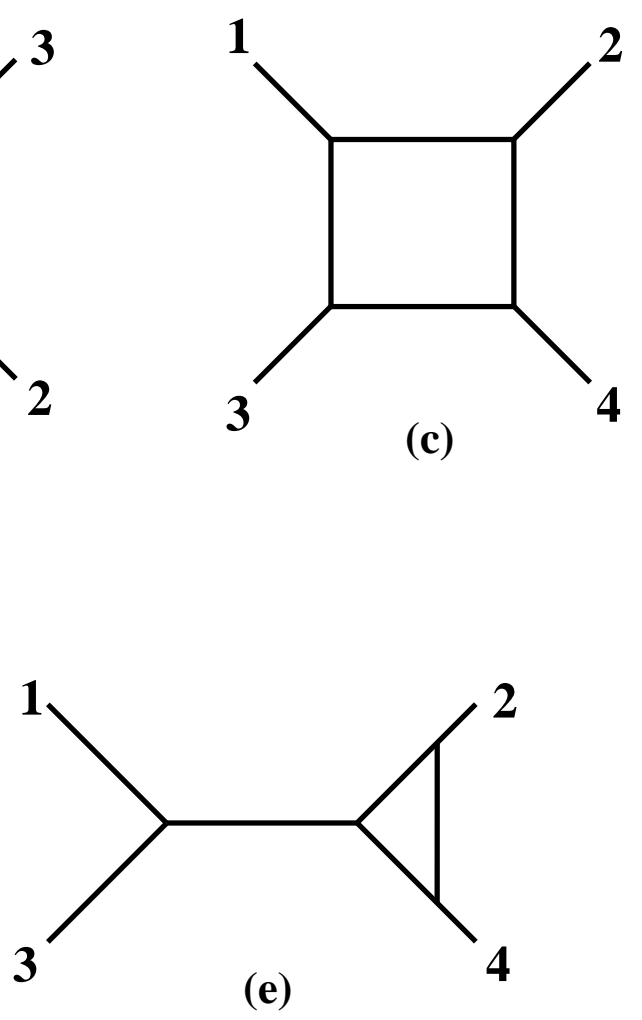

Fig. 1 